\title{
Diffusion dynamics in small-world networks with heterogeneous consumers
}

\author{
Sebastiano A. Delre \\ Faculty of Management and Organization \\ University of Groningen, e-mail: s.a.delre@rug.nl \\ Wander Jager \\ Faculty of Management and Organization \\ University of Groningen, e-mail: w.jager@rug.nl \\ Marco A. Janssen \\ School of Informatics \& \\ Center for the study of Population, Institutions and Environmental Change \\ Indiana University, e-mail: maajanss@indiana.edu
}

\begin{abstract}
Diffusions of new products and technologies through social networks can be formalized as spreading of infectious diseases. However, while epidemiological models describe infection in terms of transmissibility and susceptibility, we propose a diffusion model that explicitly includes consumer decision-making affected by social influences and word-of-mouth processes. In our computational model consumers' probability of adoption depends on the external marketing effort and on the internal influence that each consumer perceives in his/her personal social networks. Maintaining a given marketing effort and assuming its effect on the probability of adoption as linear, we can study how the speed of the diffusion depends on the network structure and on consumer heterogeneity. First, we show that the speed of diffusion changes with the degree of randomness in the network. The speed is low in regular networks, it increases in smallworld networks and finally it becomes low again in random networks. Second, we show that heterogeneity helps the diffusion. Alteris paribus and varying the degree of heterogeneity in the population of agents results show that the more heterogeneous the population, the faster the speed of the diffusion. These results contribute to marketing strategies for the launch and the dissemination of new products and technologies.

Contact:

Sebastiano A. Delre

Faculty of Management and Organization, University of Groningen,

Groningen, The Netherlands.

Tel: +31 (0)50 3634459

Email: s.a.delre@rug.nl
\end{abstract}

Key Words: innovation diffusion, threshold models, word-of-mouth, social networks, heterogeneous markets. 


\title{
Diffusion dynamics in small-world network with heterogeneous consumers
}

\author{
Sebastiano A. Delre, Wander Jager and Marco A. Janssen
}

Technological innovation drives the progress of societies. Any time a new technology, a new device, a new product appears into a society, its members have the chance to become aware of the innovation and to relate themselves to it. In western societies people encounter new inventions and technologies on a daily basis. When the innovation is a good whose consumption is individual, single consumers can decide whether to adopt it or not. The study of the diffusion patterns of new products into consumers' societies, from their launch to their successful or unsuccessful adoption, closely involves managers and marketers whose interests are in disseminating new products in the society. Recently marketers' attention has focused on the explosion of new fashions [Gladwell, 2000] and on the buzz that accompanies these explosions [Rosen, 2000]. Apparently, in highly social susceptible contexts like clothes markets, many innovations emerge from minor events that are strongly related with the dynamics of little local networks of friends. Then the new innovative fashion trend is adopted by some early adopters which are easily influenced by new trends and once the critical mass is reached, the diffusion and the number of adoptions gets at its peak. Almost all potential consumers decide to adopt and also laggards and skeptical consumers may decide to conform adopting the new product [Rogers, 1995].

The main branch of study about innovation diffusion modeling roots in the work of Bass, 1969. The Bass model formalizes the aggregate level of penetration of a new product emphasizing two processes of communication: (1) external influence via advertising and mass media, and (2) internal influence via word-of-mouth. The decision of a consumer is described as the probability to adopt the new product during time and it is assumed to depend linearly on two forces. The first force is not related to previous adopters and represents the external influence of mass media and the other force is related to the number of adopters and represents the internal influence of word-of mouth.

$$
\frac{f(T)}{(1-F(T))}=p+q F(T)
$$

where $f(T) /(1-F(T))$ is the hazard function defining the probability of a consumer to adopt at time t, p reflects the mass media influence which is independent of previous adoption and q reflects the influence due to word-of mouth. This basic Bass model fits very well to real data of durable goods and many other variations of the model have appeared in order to explain different aspects of the diffusion. (For overviews see Mahajan and Muller, 1979 and Mahajan et al. 2000) The model displays a cumulative S curve of adopters and the fast growth is generated by the social interaction between early and late adopters [Rogers, 1995]. However, the aggregate Bass model assumes all consumers to be homogeneous. It does not specify at the micro level what is the consumer decision-making during time and how consumers communicate and influence each other. One of the rare examples of micro-level models of diffusion process in a traditional economic framework is the study of Chatterjee and Eliashberg, 1990. This study presents an analytical model of innovation diffusion based on an individual decision-making that determines the adoption of agents one by one. The decision of adopting depends on the characteristics of the consumers, namely the perception of the innovation, the personal preference and the perceived reliability of information. This model allows introducing heterogeneity in the individual parameters of the population of potential consumers and these specific parameters are tested by a pilot study conducted in an experimental laboratory setting.

Although Chatterijee and Eliashberg's model represents a complete framework that links individual decisionmaking and the aggregate dynamics of innovation diffusion processes and it generates much interests on the impact of heterogeneity on diffusion models [Bemmaor and Lee, 2002], the analytical tractability of the model obliges to limited analysis of aggregated variables and of consumers characteristics. This holds both for the estimation of the parameters and in the laboratory experiments.

Another big part of the research on innovation diffusion has focused on empirical studies that show the crucial factor of the structure of social interactions [Rogers and Kincaid, 1981; Valente, 1995] and on computational models that investigate the patterns of innovation diffusion through social networks [Abrahamson and Rosenkopf, 1997; Goldenberg et al., 2000; Weisbuch and Staffeur, 2000; Young, 2002]. These models are based on the similarities between viral marketing dynamics and disease diffusions [Moore and Newman, 2000; Newman, 2002; Dodds and Watts, 2005]. However, while these epidemiological models describe infection in terms of transmissibility and susceptibility, we propose a diffusion model that explicitly includes consumer decision-making affected by social influences and word-of-mouth processes. In fact the agents of our simulation model both decide according to their preference and are influenced by other agents' decisions according to a threshold rule [Granovetter, 1978]. The model allows us to study the diffusion patterns in time for different markets. In particular, we focus our analysis on markets that differ in the social structure connecting the consumers, the strength of the social influence of the market and the heterogeneity of the consumers. 


\section{The model}

In our innovation diffusion model, all agents are connected in a unique network. The nodes of the network are the consumers and each link between two nodes represents a relation of friendship between two consumers. Such network can vary from completely regular $(\mathrm{r}=0)$ to completely random $(\mathrm{r}=1)$ [Watts and Strogatz, 1998]. On one hand, when the network is completely regular, agents are very clustered and any information takes long time in order to travel from a node to another distant node. On the other hand, when the network is completely random, agents are not clustered at all and any information is spread to all other nodes within a very short time. However, in the case in between (in the so called small-world area, $0<\mathrm{r}<1$ ), the network is both still very clustered and information spreads very fast to all the clusters of the network. The penetration of the product in the population of consumers is like an infection of a disease that propagates into this network.

The probability of agent $i$ to adopt the innovation depends on a constant external influence (marketing effort) $e_{1}$ and on an internal word-of mouth process:

$$
a_{i, j}=e_{1}+\mathrm{P}\left(U_{i, j} \geq U_{i, M I N}\right)
$$

Agents are involved in the word-of-mouth process if and only if at least one of their neighbors already adopted. In this case, they use a simple weighted utility of individual preference and social influence:

$$
U_{i j}=\beta_{j} \cdot x_{i}+\left(1-\beta_{j}\right) \cdot y_{i}
$$

where

$$
\begin{aligned}
y_{i} & =\left\{\begin{array}{l}
q_{j} \geq p_{i} \Rightarrow 1 \\
\text { otherwise } \Rightarrow 0
\end{array}\right. \\
x_{i} & =\left\{\begin{array}{l}
A_{i} \geq h_{i} \Rightarrow 1 \\
\text { otherwise } \Rightarrow 0
\end{array}\right.
\end{aligned}
$$

$U_{i, M I N}$ specifies $i$ 's minimum utility requirement and $U_{i, j}$ is the utility agent $i$ has if it adopts innovation $j$. The utility has two components that are threshold functions: individual preference $y_{i}$ and local social influence $x_{i}$ of $i$ 's personal network; $\beta_{j}$ weights these two components and it represents how strong the social influence is in the market of product $j$. Concerning the individual part, $p_{i}$ is the individual preference of agent $i$ and $q_{j}$ is the quality of the innovation $j$. Concerning the social influence $h_{i}$ is a threshold which determines the individual agent's susceptibility to its neighbors' behavior and $A_{i}$ is the fraction of adopters in the $\mathrm{L}^{\text {th }}$ order set of alters of agent $i$ (personal network) Agents included in $i$ 's personal network are called alters. Direct friends are first alters $(\mathrm{L}=1)$, friends of friends are second alters $(\mathrm{L}=2)$ and so on. If the fraction of adopters in $i$ 's personal network is higher than $h_{i}$ then the agent does feel social influence, otherwise it does not. The rationale of this formalization is the classical threshold mechanism of collective action: a consumer does not feel social pressure if just a few people around her/him behave in a particular way but once these people reach a certain number then he/she suddenly decide to change his/her mind and he/she behaves differently [Granovetter, 1978].

Diffusion is introduced in the population by external marketing effort $e_{1}$ that is assumed to be given and linear during the development of the diffusion. During all the diffusion, any non-adopter agent is convinced to adopt with probability $e_{1}$. Once an agent has adopted, other agents connected to it through their personal network become also aware of the innovation and they are involved in the word-of-mouth process evaluating their utility according to (3). Our analysis takes into consideration only a subset of the parameters' space of the model. We set $e_{1}=0.001$ and L=2 assuming that the word-of-mouth effect is more relevant than marketing effort [Goldenberg et al., 2001] and that agents can see behaviors of their friends and behaviors of their friends' friends.

In order to compare different speeds under different conditions, we report the variations in the $\rho$ indicator defined as

$$
\rho=\frac{\sum_{t=0}^{T} D(t)}{\sum_{t=0}^{T} f(t)}
$$


where $\mathrm{T}$ indicates the total cycles of the simulations, $\mathrm{D}(\mathrm{t})$ is the cumulative function of the adopters at time $\mathrm{t}$, and $\mathrm{f}(\mathrm{t})$ is the number of adopters at time $\mathrm{t}$ [Arenas et al., 2000]. The $\rho$ indicator allows us to compare different diffusions that reach the same number of adopters. Because the external marketing effort is given and constant, the speed of the diffusion is also a good indicator of how strong the word-of-mouth communication is in the market.

In our analysis we investigate how the speed of the diffusion changes when consumers have very similar or very different social sensitivity thresholds. Then we use a beta function in order to vary heterogeneity respect to the threshold $h_{i}$ of agents in the population:

$$
f(x)=\frac{x^{a-1} *(1-x)^{b-1}}{\frac{(a-1) ! *(b-1) !}{(a+b+1) !}}
$$

with mean $\mu=\frac{a}{a+b}$ varying between 0 and 1 and variance $\sigma^{2}=\frac{a^{*} b}{(a+b)^{2} *(a+b+1)}$.

Notice that the beta distribution allows us to model the heterogeneity of the agent population from the homogeneous case (very high value for $a$ and $b$ ) for which all agents have the approximately the same threshold to the uniform distribution $(a=b=1)$ for which thresholds can vary randomly around the mean value.

\section{Experimental results}

We begin investigating a very social susceptible society $\left(\beta_{j}=1, h_{i}=0.3\right)$. We varied the randomness in the network connecting the agents and observed changes in the speed of the diffusion. In Figure 1, each point represents the speed of diffusion in a network with a different degree of randomness (r).

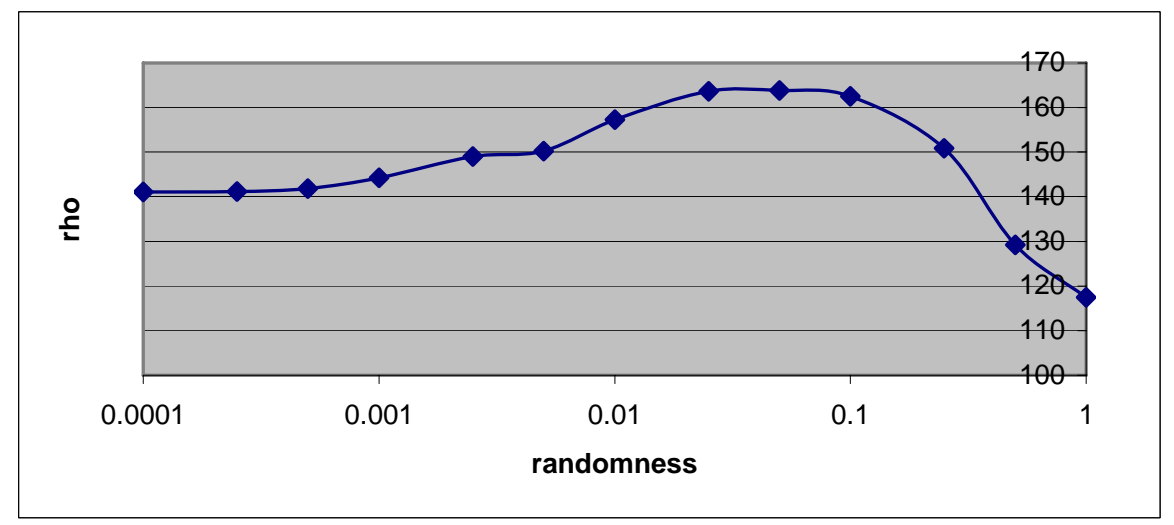

\section{Figure 1: The speed of diffusion $\rho$ (after 200 time steps) varying the degree of randomness in the network}

When the network is very clustered, a group of innovators that start the diffusion can influence only local neighbors. Such influence is strong because the more clustered the group of adopters is, the higher its influence on non-adopters neighbors becomes. Thus, the diffusion travels along the network but it cannot be spread in another distant region of the network because of the absence of shortcuts. Consequently if some agents decide to not adopt the innovation, the word-of-mouth process dies and the only way to set the diffusion process again is by external influence. Then the time needed to convince all agents of the network to adopt is relatively low. The situation changes when adding a little randomness into the network. Then shortcuts allow the innovation to emigrate in different parts of the network and diffusions can succeed easily and spread very fast. Agents can see the spreading of diffusion in other clusters and they can import the fashion in their own cluster. At the same time, social influence is still very strong because the network is very highly clustered. We observe the maximum values of $\rho$ for this smallworld area. Finally, when the randomness becomes very high, social influence is dimmed. Agents have a large number of contacts, the portion of adopters in their neighborhood is very low and there is a low internal influence on them to adopt. During the initial part of the diffusion, they may decide to adopt only because of external influence. If the external influence is low, then the critical mass will be reached very late and, only then, the rest of the population will be suddenly convinced to adopt. 
In a following set of simulations we decrease the value of $\beta_{j}$ in order to study what are the dynamics diffusion in different markets, more precisely in markets with less social influence and where consumers decide more according to their personal preferences. We set $p_{i}=[0,1]$ and $q_{j}=0.5$ assuming that agents have equal probability for positive or negative preference towards the innovation. (For an analysis of different personal preferences on hits and flops of innovations, see Delre, Jager and Janssen, 2004) We also let $h_{i}=0.3$ assuming that adopters in personal networks are relatively more visible than non-adopters.

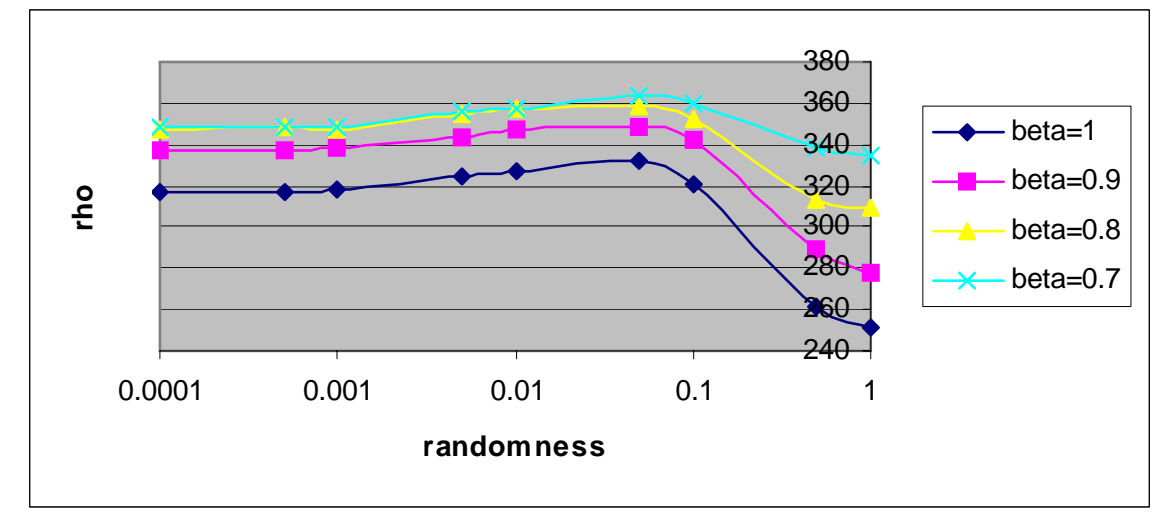

Figure 2: The speed of diffusion $\rho$ (after 400 time steps) varying $\beta_{i}$

In Figure 2 the results of this set of simulations are presented. The effects of network structures decrease when markets are less socially susceptible. Decreasing the value of $\beta_{j}$, the value of $\rho$ depends less on the topology of the social network. The small-world area (especially for $0.01<\mathrm{r}<0.1$ ) remains the most favorable for the speed of diffusion but the values of $\rho$ becomes closer to the speed of diffusion observed in more regular $(r \approx 0)$ and more random $(r \approx 1)$ networks.

In the last set of simulation runs, we include heterogeneity in the populations. With the same parameters' values as before, we observe a very high difference in the value of $\rho$ between the homogeneous case and the uniform distribution case $\left(h_{i}=0.3 \rightarrow \rho=70.408\right.$ and $h_{i}=[0,0.6] \rightarrow \rho=128.769$ after 150 time steps). Then we draw the value of $h_{i}$ from beta distributions varying the value of $a$ and $b$ in order to obtain different variance in the population that represents different degree of heterogeneity. We find that the more heterogeneous the population is, the higher the speed of the diffusion. In Figure 3, we show six S curves of diffusion for different degree of heterogeneity in the population of agents (homogeneous population, $h_{i}=0.3$; $a=12, b=28, \sigma^{2}=0.005122 ; a=9, b=21, \sigma^{2}=0.006774 ; a=6$, $b=14, \sigma^{2}=0.01 ; a=3, b=7, \sigma^{2}=0.019091$; uniform distribution $\left.h_{i}=[0,0.6]\right)$. It is evident how the time needed to complete the diffusion is much smaller as the population becomes more heterogeneous.

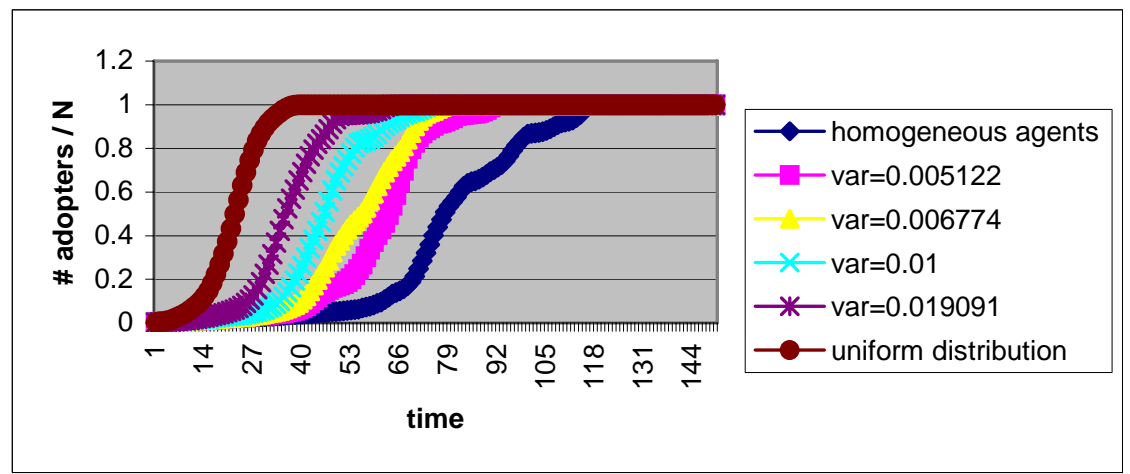

Figure 3: The S curves of diffusion varying the degree of heterogeneity in the population 


\section{Conclusions}

Markets differ in many dimensions. Here we have presented a computational model that maintains to explain innovation diffusion dynamics in markets that differ according to the social structure that connects their consumers, the strength of social influence and the degree of consumers' heterogeneity about their social susceptibility.

There are markets, like those of little towns, where consumers are very clustered. This means that if a consumer $\mathrm{A}$ is a friend of a consumer $\mathrm{B}$ and the consumer $\mathrm{B}$ is a friend of a consumer $\mathrm{C}$, then it is very likely that also $\mathrm{B}$ and $\mathrm{C}$ are friends. But there are also markets, like those of big cities, where consumers have more various kinds of relationships and such transitivity does not occur. Our model suggests that diffusions of new products and new technologies develop faster when consumers are connected in networks that are in between little towns and big cities. This means that innovations spread faster when in the groups of consumers there are both many internal relationships and enough external relationships. Many internal relationships make the influence of initial adopters on non-adopters very high and the external relationships facilitate the connections of different groups of friends.

Moreover there are very high fashionable markets in which consumers communicate and influence a lot each others and there are other markets in which consumers decide only according to their personal preferences without considering whether others decide to adopt or not. Our innovation model proposes that innovations are more likely to emerge and expand faster in markets in which consumers take heavily into consideration what others do. It is easier to launch a fashion if the diffusion is set up in high socially susceptible contexts like groups of young friends that carefully observe what other peers do.

Finally there are markets in which individual consumers differ a lot about their preferences and their social susceptibility to follow others' behaviors. In our simulation experiments we can observe that diffusions are triggered easier and faster in more heterogeneous networks of consumers. Here a few innovators ignite the diffusion sooner than in a homogeneous consumer population because they are sooner imitated by some other consumers. Consequently the critical mass is reached sooner and also the innovation can be proposed sooner to other potential consumers.

\section{References}

Abrahamson, E. and Rosenkopf, L. (1997) Social Network Effects on the Extend of Innovation Diffusion, Organization Science, 8, 289-309.

Arenas, A., Díaz-Guilera, A., Perez, C. J. and Vega-Redondo, F. (2000) Self-Organized Evolution in a Socioeconomic Environment, Physical Review E, 61, 3466-69.

Bemmaor, A. C. and Lee, Y. (2002) The impact of heterogeneity and ill-conditioning on diffusion model parameter estimates, Marketing Science, 21, 209-220.

Bass, F. M. (1969) A new product growth for model consumer durables, Management Science, 15, 215-227.

Chatterjee, R. Eliashberg, J. (1990) The Innovation Diffusion Process in the Heterogeneous Population: a Micromodelling Approach, Management Science, 36, 1057-1079.

Delre S. A. Jager, W. and Janssen, M. A., (2004) Percolation and innovation diffusion models compared: do network structures and social preferences matter? Proceedings of M2M2 Workshop and ESSA Conference, Valladolid, Spain.

Dodds, P.S. and Watts, D. J. (2005) A Generalized Model of Social and Biological Contagion, Journal of Theoretical Biology, 232, 587-604.

Gladwell, M. (2000) The Tipping Point. How Little things Can Make a Big Difference, Little Brown and Company, London.

Goldenberg, J. Libai, B. Solomon, S. Jan, N. and Stauffer, D. (2000) Marketing Percolation, Physica A, 284, 335347.

Goldenberg, J. Libai, B. and Muller, E. (2001) Talk of the Network: a Complex System Look at the Underlying Process of Word-of-Mouth, Marketing Letters, 12 (3), 211-223.

Granovetter, M. S. (1978) Threshold Models of Collective Behavior, Journal of Sociology, 83, 1420-1443.

Janssen, M. A. and Jager W. (2003) Self-Organisation of Market Dynamics: Consumer Psychology and Social Networks, Artificial Life, 9 (4).

Mahajan, V. Muller, E. (1979) Innovation Diffusion and New Product Growth Models in Marketing, Journal of Marketing, 43, 55-68.

Mahajan V. Muller, E. Wind, J. eds. (2000) New Product Diffusion Models, Kluwer Academic Publishers, Boston MA.

Moore, C. and Newman, M. E. J. (2000) Epidemics and Percolation in Small-World Networks, Physical Review E, $61,5678-82$.

Newman, M. E. J. (2002) Spread of Epidemic Disease on Networks, Physical Review E, 66, 016128.

Rogers, E. M. (1995) Diffusion of Innovation, $4^{\text {th }}$ edition, The Free Press, NY. 
Rogers, E. M. and Kincaid, D. L. (1981) Communication Networks: Toward a New Paradigm for Research, Free Press, New York

Rosen, E. (2000) The Anatomy of Buzz, Doubleday, New York.

Valente, T. (1995) Network Models of the Diffusion of Innovations, Hampton Press, Cresskill, NJ.

Watts, D. J., Strogatz, S. H. (1998) Collective dynamics of “small-world” networks, Nature, 393, 440-442.

Weisbuch, G. Stauffer, D. (2000) Hits and Flops Dynamics, Physica A, 287, 563-576.

Young, P. H. (2002) The Diffusion of Innovation in Social Networks, Santa Fe Institute working paper, 02-04-018. 